\title{
MATERIALIZACIÓN DE LOS DERECHOS SEXUALES Y REPRODUCTIVOS: ACCESO A TÉCNICAS DE REPRODUCCIÓN ASISTIDA COMO GARANTÍA DE LA SALUD Y AUTONOMÍA REPRODUCTIVA
}

\author{
Oscar Andrés Upegui Toledo* \\ Jessica Johanna Archila Julio** \\ María Angélica Otero González ${ }^{* *}$ \\ Recibido: Mayo 23 de 2016 \\ Aprobado: Junio 21 de 2016
}

\begin{abstract}
RESUMEN
El presente artículo busca exponer los lineamientos para la protección de los derechos sexuales y reproductivos, en el ámbito de la salud y la autonomía reproductiva, establecidos por la Corte Constitucional Colombiana en sentencias de tutela, así como la procedencia de esta acción para hacer efectiva la protección de los mismos. Asimismo, busca analizar el avance progresivo de las subreglas seguidas por la Corte para el acceso a Técnicas de Reproducción Asistida mediante el Sistema General de Seguridad Social en Salud, las cuales han cambiado el paradigma sobre la protección de los derechos sexuales y reproductivos.
\end{abstract}

Palabras Clave: Derecho a la salud, derechos sexuales y reproductivos, acción de tutela, tratamientos de fertilidad, Corte Constitucional, técnicas de reproducción asistida.

\section{MATERIALIZATION OF SEXUAL AND REPRODUCTIVE RIGHTS: ACCESS TO ASSISTED REPRODUCTIVE TECHNOLOGY AS A GUARANTEE OF REPRODUCTIVE AUTONOMY AND HEALTH}

\begin{abstract}
This article seeks to explain the sexual and reproductive rights protection framework in the sphere of reproductive autonomy and

*Abogado Universidad Autónoma de Bucaramanga, Unab. Correo electrónico: oupegui33@unab.edu.co ** Abogada Universidad Autónoma de Bucaramanga, Unab. Correo electrónico: jarchila5@unab.edu.co *** Abogada Universidad Autónoma de Bucaramanga, Unab. Correo electrónico: motero20@unab.edu.co
\end{abstract}


health, established by the Constitutional Court of Colombia through its amparo decisions, and the viability of this action to protect those rights. Also, it seeks to analyze the Constitutional Court regulation about Assisted Reproductive Technology access through Health Care in Colombia and its progress which has changed the paradigm about sexual and reproductive rights protection.

Keywords: right to health, sexual and reproductive rights, amparo decisions, fertility treatment, Constitutional Court, Assisted Reproductive Technology.

\section{MATERIALIZAÇÃO DOS DIREITOS SEXUAIS E REPRODUTIVOS : O ACESSO A TÉCNICAS DE REPRODUÇÃO ASSISTIDA PARA GARANTIR A SAÚDE E AUTONOMIA REPRODUTIVA RESUMO}

Este artigo procura expor as diretrizes para a proteção dos direitos sexuais e reprodutivos, no campo da saúde e da autonomia reprodutiva, estabelecidos pela Corte Constitucional da Colômbia em sentencias de "tutela", assim como a procedência desta ação para fazer efetiva a proteção dos mesmos. Portanto, este artigo procura analizar a evolução de sub- regras seguidas pela Corte para acesso a técnicas de Reprodução Assistida por meio do Sistema Geral de Segurança Social em Saúde, as quais tem mudado o paradigma sobre a protecção dos direitos sexuais e reprodutivos .

Palavras-chave: Direito à saúde, direitos sexuais e reprodutivos, tutela, tratamentos de fertilidade, Corte Constitucional, técnicas de reprodução assistida.

\section{INTRODUCCIÓN}

El avance en la jurisprudencia constitucional sobre el acceso a Técnicas de Reproducción Asistida mediante el Sistema General de Seguridad Social en Salud ha abierto una puerta para la garantía de los derechos de las personas que tienen alguna afectación en su fertilidad, lo cual les impide alcanzar un bienestar físico y social. Esto, teniendo en cuenta que la infertilidad hoy en día es catalogada como una enfermedad.

A través del presente texto, el lector podrá evidenciar la importancia del reconocimiento y la conceptualización de los derechos sexuales y reproductivos, como parte del derecho a la salud en la jurisprudencia 
constitucional colombiana, teniendo en cuenta su carácter como derechos fundamentales y el cambio de paradigma en las tesis usadas por la Corte, quien después de varios años garantiza el acceso a Técnicas de Reproducción Asistida tomando como principio el enfoque de derechos.

Por su parte, el derecho a la salud se encuentra consagrado en la Constitución Política de 1991, así como en números instrumentos institucionales, y actualmente es un derecho autónomo, con carácter fundamental (Sentencia T-760, 2008) sin embargo el reconocimiento de los derechos sexuales y reproductivos no ha sido el mismo. Considerando que en la Convención para la eliminación de todas las formas de discriminación contra la mujer (CEDAW, 1979) se hace alusión a la planificación familia y al parto en sus artículos, no se ha hecho una inclusión taxativa en referencia a los derechos sexuales y reproductivos que, por cierto, no conciernen únicamente a las mujeres, relacionándose también con el hombre y las afectaciones reproductivas que puedan llegar a tener.(Gherardi, 2011).

También en la Corte Interamericana de Derechos Humanos se ha dejado entrever una fuerte relación de los derechos sexuales y reproductivos con la autonomía personal, la libertad reproductiva, la integridad física y sicológica. Dicho tribunal ha establecido que:

Los derechos a la vida privada y a la integridad personal se hallan también directa e inmediatamente vinculados con la atención de la salud. La falta de salvaguardas legales para tomar en consideración la salud reproductiva puede resultar en un menoscabo grave del derecho a la autonomía y la libertad reproductiva. Existe por tanto una conexión entre la autonomía personal, la libertad reproductiva y la integridad física y psicológica. (Caso Artavia Murillo y otros Vs. Costa Rica, 2012, pág. 47).

En el mismo caso, la Corte Interamericana de Derechos Humanos ha establecido en relación a los derechos sexuales y reproductivos, analizados desde la óptica de la libertad del ser humano de reproducirse, lo siguiente:

La mujer y el hombre están en libertad para decidir si desean reproducirse y en qué momento, y tienen el derecho de estar informados y tener acceso a métodos de planificación familiar seguros, eficaces, asequibles y aceptables de su elección, así como el derecho de acceso a 
los pertinentes servicios de atención de la salud. (Comité de Derechos Económicos, Sociales y Culturales, 2000, pág. 12).

En ese orden de ideas, los Derechos Sexuales y Reproductivos han sido reconocidos y protegidos, de acuerdo a los criterios de "(i) la autodeterminación reproductiva y (ii) el acceso a servicios de salud reproductiva" (Sentencia T-627, 2012). Se debe resaltar que los derechos materia de estudio en este artículo, encuentran conexidad no sólo con el derecho a la salud sino que además se ven relacionados con otros derechos fundamentales como lo son el derecho a la vida digna, a la igualdad, al libre desarrollo de la personalidad, a la educación y la información como ha sido reiterado en jurisprudencia de la Corte. Siguiendo los lineamientos de la Corte, es posible afirmar que las Técnicas de Reproducción Asistida (TRA) son una garantía para que efectivamente se materialice el ejercicio de los derechos sexuales y reproductivos, en conexidad con el derecho a la salud, específicamente para personas con infertilidad por considerarse servicios médicos que ayudan a superar la barrera de la infertilidad (Sentencia T-528, 2014).

Teniendo en cuenta lo anterior, se procederá a analizar cuál ha sido la postura establecida por la Corte Constitucional en casos similares, si se ha garantizado o no los derechos sexuales y reproductivos analizados como derechos fundamentales, si su protección y garantía son obligaciones inmediatas para el Estado. Esto, con el fin de generar una argumentación solida por la cual las TRA materializan de manera efectiva los derechos sexuales y reproductivos de las personas que tienen alguna afección en su salud, en este caso, la infertilidad, ya sea primaria o secundaria, lo cual redunda en una mejor calidad de vida de los individuos.

El presente artículo, busca establecer la existencia de una garantía a nivel gubernamental que permita acceder a las técnicas de reproducción asistida a través del Sistema de Seguridad de Salud Social en Colombia. Para ello, se realizó un análisis documental de tipo cualitativo la jurisprudencia de la Corte Constitucional que toman como eje central temas de la salud sexual y reproductiva, el derecho a la salud, derecho a la familia y lo pertinente a técnicas de reproducción asistida, haciendo una recopilación desde el año 2000 hasta el año 2015 finalizando con la sentencia hito T-274 del mismo año, asimismo, se revisó la doctrina y ordenamiento jurídico relevante. Para el análisis jurisprudencial se utilizó el método de análisis dinámico de jurisprudencia diseñado por Diego López Medina en su libro el Derecho de los jueces. El análisis 
presentado, permitirá dar una nueva perspectiva a la protección de Derechos humanos fundamentales como son los derechos sexuales y reproductivos, dando como conclusión la procedencia de la acción de tutela para acceder a este tipo de técnicas.

\section{GARANTÍA DE PROTECCIÓN DE LOS DERECHOS SEXUALES Y REPRODUCTIVOS: DERECHO A LA MATERNIDAD Y PATERNIDAD}

Como objeto de análisis de este artículo, se tomarán las Técnicas de Reproducción Asistida, como mecanismo alternativo para solucionar problemas relacionados con la infertilidad ya sea primaria o secundaria, que de acuerdo a la Organización Mundial de la Salud (OMS) está definida como "una enfermedad que afecta la salud física y mental de la persona que la sufre, afectando también a su pareja y la esfera que la rodea. Se puede llegar a identificar esta dolencia, cuando después de haber mantenido relaciones sexuales por más de 12 o 24 meses sin protección, no es posible concebir un hijo, cada caso es particular y puede ser originado por múltiples causas en la mujer o el hombre" (Cook, Dickens, \& Fathalla, 2003).

Es importante mencionar, que se habla de una distinción, entre la infertilidad originaria o primaria y la infertilidad secundaria. Se habla de infertilidad primaria cuando ésta origina la incapacidad de concepción de un hijo de manera biológica, y la infertilidad secundaria es aquella que surge como consecuencia de otra enfermedad; este concepto es relevante cuando la Corte Constitucional decide determinar la procedencia de la acción de tutela tomando como base el origen de la enfermedad y si causa en la persona daños irremediables a su salud física y/o vida. Siendo la infertilidad secundaria la más opcionada para recibir tratamientos médicos, ya que al no tratarse de una enfermedad base u originaria, puede ser tomada como una doble afectación a la vida e integridad de las personas por lo cual se debe procurar su bienestar; así como lo ha sostenido la Corte en sentencias donde se relaciona como un presupuesto para garantizar el acceso a las TRA. (Sentencia T-890, 2009). Al respecto la Corte ha expresado que:

La infertilidad es una enfermedad que afecta el sistema reproductivo y que interfiere con la capacidad, temporal o permanente, de una pareja heterosexual para alcanzar un embarazo, a pesar de mantener una vida sexual activa por más de un año y sin control anticonceptivo voluntario. 
Por su parte, la infertilidad secundaria hace referencia generalmente- a aquellos pacientes que tienen antecedentes de uno o varios embarazos y luego de un tiempo les resulta imposible concebir. En las mujeres las principales causas de este tipo de infertilidad son los problemas ováricos, ovulación deficiente, obstrucción no genética de las trompas de Falopio, alteraciones hormonales o enfermedades infecciosas que son transmitidas sexualmente; en el hombre, anomalías repentinas en la producción hormonal. Así, la infertilidad secundaria es causada por otro tipo de afecciones físicas o enfermedad autónoma que limitan la capacidad de una persona para engendrar y solo en esos casos merecen una protección excepcional por vía de tutela.(Sentencia T-946, 2007).

Es así, como el uso de la fecundación asistida es una alternativa viable para combatir la infertilidad, y se encuentra en conexidad con el goce de los beneficios del progreso científico, derecho que ha sido reconocido internacionalmente en el artículo 15 literal b del Pacto Internacional de Derechos Económicos, Sociales y Culturales, PIDESC (Naciones Unidas, 1976).

Teniendo en cuenta el grado de oportunidad de las personas que pueden acceder de manera efectiva a este tipo de procedimientos en Colombia, no se podría hablar de una garantía al derecho de maternidad y de paternidad, cuando se trata de una exclusión taxativa del POS (Resolución 1479, 2015), en razón a que son procedimientos que requieren una alta inversión económica y no existe un consenso general sobre su accesibilidad mediante el Sistema de Salud colombiano, debido a que una inversión en estos procedimientos podría generar un menoscabo en la efectiva prestación de otros tratamientos que se han incluido desde hace tiempo en el plan de salud colombiano.

Existen casos en que la fertilidad se muestra como un obstáculo para el libre desarrollo del derecho a la maternidad y la paternidad, derecho que implica además de la reproducción biológica, que esta se dé en circunstancias adecuadas, seguras y necesarias para su salud tanto de la madre como del hijo que está por nacer.

Históricamente ha sido notoria y general que la responsabilidad de la procreación recae sobre la mujer, pues biológicamente se encuentra diseñada para cumplir con la 
función reproductiva, por esta razón las TRA están siendo usadas en gran parte por mujeres que buscan ser beneficiadas por este tratamiento. Si bien es cierto, la pareja comparte la responsabilidad de la infertilidad, pues las causas de la infertilidad pueden provenir de cualquiera, ya sea hombre o mujer; por razones biológicas y sociales, la carga de la infertilidad no se comparte de manera equitativa. Es así como la investigación de las causas de infertilidad es más exhaustiva en la mujer que en el hombre y asimismo, está asociada con mayores inconvenientes y riesgos para ella. (Cook, Dickens, \& Fathalla, 2003 p. 41)

Legalmente no ha sido posible establecer el libre acceso a las técnicas de reproducción asistida, así como tampoco se han encontrado normas que contraríen la posibilidad de ejercer el derecho a la maternidad o la paternidad porque el entorno laboral o social haga difícil el cumplimiento de esta tarea. Es así como al enfrentarse ante la imposibilidad de no ejercer libremente su derecho a la maternidad o paternidad, ven afectado su derecho al libre desarrollo de la personalidad.

El optar por la maternidad, la paternidad o la conformación de una familia, se debe proteger dentro del marco del derecho al libre desarrollo de la personalidad, como se ha mencionado. No es legítimo establecer reglas que desestimulen la maternidad o la paternidad, por eso la legislación laboral protege mediante la licencia de maternidad y paternidad espacios para la configuración del vínculo materno y paterno filial, de los hijos recién nacidos y los trabajadores. (Pabón Mantilla \& Aguirre Román, 2009).

\section{INCLUSIÓN DE LOS DERECHOS SEXUALES Y REPRODUCTIVOS EN EL SISTEMA GENERAL DE SEGURIDAD SOCIAL EN SALUD DE COLOMBIA}

Como antecedente histórico, vemos que el debate sobre derechos sexuales y reproductivos inicia en 1948 en la Declaración Universal de Derechos Humanos en el marco de las Naciones Unidas, más adelante en 1966 los Pactos Internacionales de Derechos Civiles y Políticos y de Derechos Económicos Sociales y Culturales (PIDESC) dieron más contenido a estos derechos e indicaron los deberes y las responsabilidades de los Estados frente a los particulares, definiendo mecanismos para verificar su cumplimiento. 
En Colombia, se ha dado de manera paulatina y tardía la protección a los derechos sexuales y reproductivos a través de la Jurisprudencia de la Corte Constitucional, así como también a través del bloque de constitucionalidad se ha hecho su inclusión a partir del enfoque de derechos con carácter fundamental, por encontrarse protegidos por la legislación internacional referente a derechos humanos y por otros documentos de las Naciones Unidas; entre los principales instrumentos legales internacionales que los apoyan están: Declaración Universal de los Derechos Humanos (1948); Pacto Internacional de Derechos Civiles y Políticos (1976); Pacto de Derechos Económicos y Sociales (1976); Convención Internacional para la Eliminación de Todas las Formas de Discriminación contra la Mujer (1981); Convención sobre los Derechos del Niño (1990), Declaración y Programa de Acción de la Conferencia Internacional de Derechos Humanos, Viena (1993), Programa de Acción de la Conferencia Internacional de Población y Desarrollo, El Cairo (1994), Programa de Acción de la Conferencia Mundial de la Mujer, Beijing (1995).(Colombia Aprende)

Por iniciativa gubernamental, existe una política Nacional de Sexualidad, Derechos Sexuales y Derechos Reproductivos desde el año 2010 (Ministerio de Salud y Protección Social, 2010), sin embargo en la misma no se aborda el tema de la Reproducción asistida, ni tampoco se habla del acceso a los tratamientos de infertilidad, el propósito de este plan es la atención primaria en Salud y tiene un enfoque sobre el conocimiento y aprehensión de los conceptos y los derechos inherentes a la salud sexual y reproductiva, para que en ejercicio de su ciudadanía conozcan sus derechos y los exijan, en términos de bienestar para las personas.

Los derechos sexuales y reproductivos representan un pilar fundamental para el ejercicio de la ciudadanía, siendo esta además la posibilidad de hombres y mujeres de tomar decisiones sobre su propio cuerpo en los aspectos de sexualidad y reproducción. (Instituto Interamericano de Derechos Humanos.)

Es así como el reconocimiento de los derechos sexuales y reproductivos surge como respuesta a movimientos de lucha y reivindicación de dos grupos históricamente discriminados: las mujeres y las personas lesbianas, gays, bisexuales, trans e intersex (LGBTI); ya que es una forma de expresión de la libertad sexual y reproductiva de la que se supone un ciudadano está en condición de ejercer estos derechos, así como goza y disfruta otros derechos humanos fundamentales, teniendo en cuenta que se trata de ir más allá del solo ejercicio de estos derechos y 
su vínculo con otros derechos fundamentales, se trata también de ejercerlos de manera plena y segura, existiendo una protección al ciudadano dependiendo de su libre escogencia y de sus necesidades propias como ser humano.

Lo anterior presupone una materialización de los derechos reclamados a través de mecanismos idóneos encargados de velar por la protección de los derechos humanos fundamentales otorgados por la Constitución para todos los ciudadanos, como lo es la acción de tutela.

\section{ACCESO A LAS TÉCNICAS DE REPRODUCCIÓN ASISTIDA COMO MECANISMO DE PROTECCIÓN DE DER ECHOS FUNDAMENTALES EN LOS PRONUNCIAMIENTOS DE LA CORTE CONSTITUCIONAL COLOMBIA}

En Colombia se inició una discusión en relación al acceso a las técnicas de reproducción asistida, a pesar de que existe una prohibición taxativa en la Resolución 1479 de 2015 del Plan Obligatorio de Salud. No se ha dejado de lado la posibilidad de inclusión de tratamientos de fertilidad como forma de protección de los derechos sexuales y reproductivos; sin embargo, el obstáculo que sobresale es de carácter económico, al señalar que una inclusión de este tipo traería graves consecuencias fiscales. Sin bien es cierto, no es posible equiparar derechos humanos fundamentales con bienes de carácter económico. Así como tampoco es factible comparar afectaciones a la salud, y en caso de no encontrarse en riesgo la vida, la protección no siempre es garantizada.

Es así como la Corte Constitucional ha negado en reiteradas ocasiones en su jurisprudencia, la protección invocada a través de la acción de tutela cuando con ella se pretende la autorización de los tratamientos de fertilidad, así como es expuesto en la sentencia de tutela T-274 del año 2015.

Iniciando el análisis jurisprudencial en el año 2000 con la sentencia T1104, sobre el acceso a técnicas de fertilidad, se reconoce la relación que ciertos derechos prestacionales como lo es la seguridad social pueden llegar a tener con la vida; estableciendo que la infertilidad no pone en riesgo la vida misma, razón suficiente para negar la protección constitucional solicitada.

Posteriormente en casos analizados por la Corte en sentencias T-689 de 2001, T-946 de 2002, T-752 de 2007, bajo el mismo argumento, negó la 
protección invocada que denegó el amparo, aduciendo las siguientes consideraciones:

(i) La patología de la accionante, aparte de la incapacidad para procrear, no conlleva una afectación grave en su salud o en su vida.

(ii) La exclusión del POS de los tratamientos de fertilidad "no solo constituye el legítimo desarrollo de una facultad de configuración legal, sino que es totalmente coherente con la necesidad de implementar un Sistema de Seguridad Social en Salud que se atenga al principio de universalidad y a su garantía a todos los habitantes del territorio colombiano". Aunado a ello, si bien la Constitución protege a la mujer durante el embarazo y después del parto, "ese deber de asistencia y protección opera siempre que la procreación sea posible y solo impone el deber de no obstruir o limitar el derecho de la mujer a procrear". (Corte Constitucional, Sentencia T-274 de 2015).

Aunado a lo anterior, se reitera que la acción de tutela no debe ser utilizada como una extensión del Plan Obligatorio de Salud, cuando se trata de servicios excluidos del mismo, además señaló que si lo que se busca es la procreación biológica, existe la posibilidad de hacer uso del mecanismo legal y constitucional de la adopción, y si bien estos procedimientos pueden generar el derecho a conformar una familia y ser madre o padre, claramente no están cubriendo los derechos sexuales y reproductivos. Si bien se cumple con el deseo de ser madre, padre o de conformar una familia, no se estaría haciendo bajo el amparo de derechos reproductivos y de manera biológica como lo es el anhelo de las personas que buscar acudir a los tratamientos de fertilidad.

Más adelante en la sentencia T-009 de 2014, la Corte fue reiterativa con los parámetros constitucionales establecidos acerca del acceso a la fertilización in vitro a través del sistema de salud y agregó los siguientes argumentos:

(i) la no inclusión de dicho procedimiento en el POS constituye un legítimo desarrollo de la facultad de configuración del legislador; (ii) así un médico, aunque esté adscrito a la EPS respectiva, haya prescrito dicho procedimiento, no es per se viable otorgar tutela porque el 
derecho a ser madre y la maternidad asistida tienen límites razonables y justificados constitucionalmente; y (iii) el Estado no está obligado a apoyar y sufragar procedimientos científicos especiales, incluyéndolos en los planes obligatorios de salud, para garantizar la procreación y suplir la infertilidad.

De manera excepcional, se ha concedido la protección invocada a través de la acción de tutela cuando se solicita la autorización de tratamientos de fertilidad excluidos del Plan Obligatorio de Salud, en casos donde se vulnera el principio de continuidad en la prestación del servicio de salud, como se estableció en la sentencia T-572 de 2002:

En esa oportunidad concedió la protección invocada al considerar que, aunque existen precedentes jurisprudenciales que declararon improcedente la acción de tutela para extender la cobertura del POS a operaciones relacionadas con tratamientos de fertilidad, en este caso se presentaban dos circunstancias nuevas: (i) se trató de la entrega de medicamentos y no de procedimientos quirúrgicos; y (ii) había un tratamiento médico en curso. Sobre este último punto, señaló que "romper la continuidad del tratamiento de manera abrupta ocasiona un perjuicio irremediable y vulnera los derechos a la dignidad, a la igualdad, a la integridad física, a la confianza legítima y a la posibilidad de ser madre.

Anterior a la garantía de los derechos sexuales y reproductivos por vía jurisprudencial, la Corte había establecido que:

Por regla general, deben negarse aquellas acciones de tutela interpuestas con la finalidad de obtener la autorización de los tratamientos de fertilidad. Sin embargo, ha aceptado la procedencia excepcional de este mecanismo constitucional al menos en dos eventos (i) cuando con ello se pretende garantizar la continuidad en la prestación del servicio de salud; y (ii) cuando se busca garantizar la vida, la salud o la integridad personal, incluyendo la salud sexual y reproductiva, en los casos en los que se requiere: a) la práctica de exámenes o procedimientos diagnósticos necesarios para precisar una condición de salud asociada a la infertilidad; b) el suministro de un medicamento; y c) la 
práctica de tratamientos integrales en pacientes que padecen una enfermedad que afecta su aparato reproductor. (Sentencia T-274, 2015).

Así las cosas, se han hecho excepciones en casos donde el objetivo principal del tratamiento es buscar la recuperación de la salud del paciente, es decir en casos donde se encuentra en riesgo la vida. Por lo anterior, podemos concluir que la protección otorgada por la Corte en sede de revisión de tutela, nunca se había tomado a partir del enfoque de derechos sexuales y reproductivos como un tratamiento para la infertilidad, sólo estaba siendo tomada la patología que causaba una afección grave a la vida de la persona, sin tener en cuenta su capacidad reproductiva ni con el ánimo de incidir de manera positiva en la fertilidad de la persona que busca proteger y salvaguardar los derechos sexuales y reproductivos. Es por esa razón que la Corte ha negado el reconocimiento de tratamientos de reproducción asistida cuando su finalidad principal es la de facilitar la capacidad procreación biológica a través de los avances científicos que proporcionan una solución a la barrera de la infertilidad.

Recientemente en la sentencia T-274 de 2015, se ha cambiado el paradigma establecido por la Corte Constitucional. Se realiza en concreto el estudio de los expedientes T-4.492.963; T-4.715.291; T4.725.592; y T-4.734.867, todos ellos casos similares de personas que debido a problemas de fertilidad no podían concebir hijos sin la ayuda de una técnica de fertilidad.

En este caso, la Corte concedió el amparo a las técnicas de fertilidad teniendo en cuenta las subreglas preestablecidas por ella misma, como lo son la prestación continua del servicio de salud y la protección efectiva al derecho a la salud y a los derechos sexuales y reproductivos. De igual modo, exhortó al gobierno nacional para realizar un estudio del presupuesto asignado a la salud y así abrir la posibilidad de incluir las Técnicas de Reproducción Asistida dentro del POS sin que esto causare un detrimento en los demás tratamientos médicos que ya se encuentren incluidos en el Plan Obligatorio de Salud contemplados para la protección a la vida y garantía de los derechos humanos fundamentales que se llegaren a ver vulnerados. (Sentencia T-274 de 2015)

Constata la Sala entonces que aunque la conservación del equilibrio financiero constituye un argumento válido para la exclusión de los tratamientos de reproducción asistida del 
Plan Obligatorio de Salud, ello no significa que no se pueda avanzar en su inclusión. Además, porque resulta constitucionalmente problemático que tal proceder, esto es, mantener tales tratamientos por fuera de la cobertura del POS, sea de carácter generalizado; es decir, a pesar de ser razonable tal exclusión del plan de beneficios, hacerlo en todos los casos puede conducir a la vulneración de ciertos derechos fundamentales (...) (Sentencia T-274 de 2015)

Con este último pronunciamiento la Corte ha dejado en claro su intención de proteger los derechos sexuales y reproductivos de los ciudadanos y la inminente necesidad que existe sobre manejar de la mejor forma el déficit existente en el presupuesto de la salud para que los tratamientos que buscan proteger otro tipo de derechos como lo son los sexuales y reproductivos en conexidad a la maternidad o paternidad y otros derechos fundamentales, también sean garantizados por el Sistema General de Seguridad Social en Salud colombiano.

El tema presupuestal y las falencias que en torno a este se puedan dar y que fueron análisis de la Corte, se unen también al análisis que en esta última sentencia se hace sobre el alcance que tienen los derechos sexuales y reproductivos, se han ido reconociendo en el ámbito internacional como Derechos Humanos, y en Colombia aunque no se tienen la infertilidad como una afectación propia contra la vida, empieza a existir la necesidad de darle al tema mayor importancia.

A pesar del reconocimiento que a nivel internacional se ha introducido respecto de la infertilidad como una enfermedad, en Colombia no se le ha dado tal connotación, lo que a juicio de la Sala supone una barrera para el acceso a los servicios de salud reproductiva, e incluso limita el alcance de otros derechos fundamentales como la igualdady la integridad personal, física, psíquica y social. Como se expuso, aunque esta enfermedad no involucra gravemente la vida, la dignidad o a la integridad personal del paciente, sí podría llegar a interferir negativamente en otras dimensiones vitales desde el punto de vista del bienestar sicológico y social. (Sentencia T- 274 de 2015)

Es por lo mencionado que se entiende que la Corte ha buscado darle relevancia a la afectación que un deterioro en la salud reproductiva puede generar en la vida y la salud en el sentido amplio de las personas y 
de ahí la necesidad de una intervención efectiva del estado como protector de los ciudadanos.

\section{CONCLUSIONES}

El tema de los derechos sexuales y reproductivos en Colombia comenzó siendo algo a lo cual no se le daba importancia o relevancia alguna, pero la Corte Constitucional se ha encargado de ahondar cada vez más en estos y aunque no se haya llegado al punto de que se tengan como derechos vinculados a la vida misma de la persona, se han logrado ligar con derechos tan importantes y fundamentales como lo son la dignidad humana y la igualdad.

Un hombre, mujer o pareja, puede llegar a ver afectada su vida por diversas razones y como se estudió en el presente artículo los derechos sexuales y reproductivos han tomado gran importancia en la salud de las personas, lo anterior, articulado a la necesidad que se puede llegar a sentir por valer los derechos a la maternidad y paternidad.

Todo lo anterior está relacionado con el derecho a la salud de las personas y las afectaciones que a esta se puedan generar cuando existen padecimientos como lo es la infertilidad que aunque como ya se estableció, no genera una afectación directa a la vida, sí lo hace a la salud en el sentido amplio de esta, es decir a la salud tanto física como social y mental de las personas según ya lo reconoció la Organización Mundial de la Salud.

La Corte Constitucional colombiana, en su búsqueda por ampliar el sentido de los derechos de los ciudadanos se encargó de construir a través del tiempo una línea jurisprudencial en razón a los derechos sexuales y reproductivos de las personas y el acceso a las Técnicas de Reproducción Asistida como garantía de ellos, y como se observó en el análisis realizado a esta línea, el avance que se ha generado en el tema, aunque no es total, ha creado ciertos parámetros que en la actualidad discusiones que favorecen a las personas que se han visto afectadas en esta temática

Se ha implementado un concepto ligado a las tendencias y normas internacionales ampliando la protección que ha existido en Colombia que aunque como ya se mencionó no ha sido muy amplia, la Corte estableció en su análisis del tema vía jurisprudencia, ciertos parámetros, como lo son la continuidad del servicio y la garantía de otros 
tratamientos prescritos para proteger la salud, que al cumplirse generan una facilidad de protección a derechos reproductivos y acceso a estos mediante mecanismos como lo son las Técnicas de Reproducción Asistida a través del mecanismo idóneo para proteger los derechos fundamentales otorgado por la Constitución como lo es la tutela.

Finalmente se encuentra el tema presupuestal, pues si bien es claro según la Corte que en Colombia los recursos muchas veces son limitados y más en un tema que muchas veces requiere una alta inversión como lo es la salud, no se puede dejar de lado la búsqueda por proporcionar los gastos que se destinan a ello, tratando de equilibrar este presupuesto con el fin de que se puedan generar aportes para tratar el tema de la infertilidad y así proteger los derechos sexuales y reproductivos de las personas, que según el análisis realizado, pueden ser tan importantes como otros derechos y tratamientos que en la actualidad sí se encuentran directamente permitidos en el Plan Obligatorio de Salud del país.

\section{REFERENCIAS BIBLIOGRÁFICAS}

Caso Artavia Murillo y otros Vs. Costa Rica (Corte Interamericana de Derechos Humanos 28 de Noviembre de 2012).

Colombia Aprende. (s.f.). Derechos e hilos conductores. Obtenido de http://www.colombiaaprende.edu.co/html/productos/1685/articles172254_recurso_1.pdf

Comité de Derechos Económicos, Sociales y Culturales. (11 de Agosto de 2000). Observación General No. 14 (2000), El derecho al disfrute del más alto nivel posible de salud (artículo 12 del Pacto Internacional de Derechos Económicos, Sociales y Culturales).

Cook, R., Dickens, B., \& Fathalla, M. (2003). Salud reproductiva y Derechos Humanos: Integración de la medicina la ética y el derecho.

Gherardi, N. (2011). La Justicia en Construcción. Buenos Aires: Equipo latinoamericano de Justicia y Género.

Instituto Interamericano de Derechos Humanos. (s.f.). Guía de capacitación en derechos humanos en las mujeres. . Obtenido de Tejiendo el cambio: http://www.iidh.ed.cr/BibliotecaWeb/Varios/ Documentos.Interno/ 
Ministerio de Salud y Protección Social. (20 de Septiembre de 2010). Biblioteca digital del Ministerio de Salud. Obtenido de https://www.minsalud.gov.co/sites/rid/Lists/BibliotecaDigital/RIDE/D E/LIBRO\%20POLITICA\%20SEXUAL\%20SEPT\%2010.pdf

Naciones Unidas. (1976). Pacto Internacional de Derechos Económicos, Sociales y Culturales PIDESC.

Pabón Mantilla, A. P., \& Aguirre Román, J. O. (2009). La Protección Jurisprudencial de la Maternidad en Colombia. Diálogos de saberres: Investigaciones y Ciencias Sociales.

Resolución 1479. (2015). Sistema del Plan Obligatorio de Salud. Colombia.

Sentencia T-274, T-274 (Corte Constitucional de Colombia 2015).

Sentencia T-528, T-528 (Corte Constitucional de Colombia 18 de Julio de 2014).

Sentencia T-627 (Corte Constituciona 10 deAgosto de 2012).

Sentencia T-627, T-627 (Corte Constitucional de Colombia 10 de Agosto de 2012).

Sentencia T-760, T-760 (Corte Constitucional de Colombia 31 de Julio de 2008).

Sentencia T-890, T-890 (Corte Constitucional 2009).

Sentencia T-946, T-946 (Corte Constitucional de Colombia 2007). 